\title{
FOCO NOS NEGÓCIOS X FOCO NOS \\ INDIVÍDUOS: RAZÕES PARA IMPLEMENTAR \\ PRÁTICAS DE RESPONSABILIDADE \\ EMPRESARIAL NO TURISMO BRASILEIRO
}

\section{BUSINESS FOCUS VS. INDIVIDUAL FOCUS: REASONS FOR IMPLEMENTING CORPORATE RESPONSIBILITY IN THE BRAZILIAN TOURISM SECTOR \\ FOCO EN LOS NEGOCIOS X FOCO EN LOS INDIVIDUOS: RAZONES PARA IMPLEMENTAR PRÁCTICAS DE RESPONSABILIDAD EMPRESARIAL EN EL TURISMO BRASILEÑO}

\section{Helena Araújo Costa}

Professora Adjunta IV da Universidade de Brasília. Líder do LETS - Laboratório de Estudos em Turismo e Sustentabilidade/UnB.

Doutora em Desenvolvimento Sustentável (CDS/UnB)

helenacosta@unb.br

Orcid: https://orcid.org/0000-0002-5076-7977

\section{Xavier Font}

Professor de Marketing e Sustentabilidade na University of Surrey, Reino Unido. Coordenador do Sustainability and Well-being Research Group/University of Surrey.

Co-Diretor do International Centre for Responsible Tourism (ICRT).

PhD in Sustainable Tourism Certification (Leeds Beckett University).

$$
\text { x.font@surrey.ac.uk }
$$

Orcid: https://orcid.org/0000-0003-1770-6213

\section{Lluís Garay}

Professor Senior do Departamento de Economia e Gestão da Universitat Oberta de Catalunya (UOC).

Coordenador do grupo de pesquisa NOUTUR - Novas Perspectivas em Turismo e Lazer e Diretor do Programa de Mestrado em Responsabilidade Social Corporativa/UOC. Phd pela Universitat Autònoma de Barcelona.

$$
\text { lgaray@uoc.edu }
$$

Orcid: https://orcid.org/0000-0002-4209-3319

\section{Steve Jones}

Professor na Escola de Eventos, Turismo e Hospitalidade da Leeds Beckett University, Reino Unido. 
s.r.jones@leedsbeckett.ac.uk

Orcid: https://orcid.org/0000-0001-6815-2957

Data de submissão: 08/11/2017 - Data de aceite: 13/04/2018

RESUMO: O objetivo do estudo foi identificar o comportamento responsável de empresas de turismo no Brasil e seus motivos para implementar tais ações. A pesquisa obteve 1.350 respostas de empresas de turismo pertencentes a diversos segmentos, tais como alojamento, viagens, alimentação e transporte turístico. A metodologia foi composta por coleta de dados quantitativa via internet, seguida de interpretação baseada em estatística descritiva e análise de clusters. Os resultados indicaram que, entre as empresas de turismo do Brasil, as medidas ambientais são as mais frequentemente implementadas, com destaque para aquelas voltadas para a economia de energia ou água. A análise evidenciou dois grupos distintos, respaldados pelo referencial teórico, quando se trata das razões para implementar as práticas responsáveis: um com foco nos negócios e outro com foco nos indivíduos. Enquanto o primeiro apresenta razões inerentes ao negócio, tais como redução de custos e ampliação da competitividade; o outro grupo tem motivos ligados ao modo de vida e aos valores do indivíduo. O grupo, que tem motivações centradas no indivíduo, revelou-se maior e com mais implementações das medidas investigadas nos três pilares da sustentabilidade. Por fim, não houve constatação de diferenças no desempenho financeiro entre empresas dos dois grupos.

PALAVRAS-CHAVES: responsabilidade corporativa, turismo, Brasil.

ABSTRACT: This study aims to identify the responsible actions taken by tourism businesses in Brazil, focusing on the reasons for implementing such actions. The survey obtained 1,350 responses from tourism companies in sectors such as accommodation, travel agencies, food and transportation. The methods consisted of an online survey, and the data analysis was performed using descriptive statistics and cluster analysis. The results indicate that among the tourism companies in Brazil, environmental measures are the measures most frequently implemented, particularly those aimed at saving energy or water. The analysis revealed two distinct groups in terms of the reasons for implementing responsible practices, both supported by the theoretical framework. One was focused on the business, and the other on individuals. While the first group presented reasons focused on the business, such as cost reduction and increased competitiveness, the second group presented motivations related to the individual, such as lifestyle and personal values. The individual motivations group was larger, and implemented more of the measures investigated in the triple bottom line of sustainability. Finally, there was no evidence of differences in financial performance in the companies of the two groups.

KEYWORDS: corporate responsibility, tourism, Brazil.

RESUMEN: El objetivo del estudio fue identificar el comportamiento responsable de empresas de turismo en Brasil y sus motivos para implementar tales acciones. La investigación obtuvo 1.350 respuestas de empresas de turismo pertenecientes a diversos segmentos, tales como alojamiento, viajes, alimentación y transporte turístico. La metodología fue compuesta por la recolección de datos cuantitativo vía internet, seguido de interpretación basada en estadística descriptiva y análisis de clusters. Los resultados indicaron que, entre las empresas de turismo en Brasil, las medidas ambientales son las más frecuentemente implementadas, con destaque para aquellas orientadas para la economía de energía o agua. El análisis demostró dos grupos distintos, respaldados por el referencial teórico, cuando se trata de las razones para implementar las prácticas responsables: uno con foco en los negocios y otro con foco en los individuos. Mientras, el primero presenta razones inherentes al negocio, tales como reducción de costos y ampliación de la competitividad; el otro grupo tiene motivos ligados al modo de vida y a los valores del individuo. El grupo, que tiene motivaciones centradas en el individuo, se revelo mayor y con más implementaciones de las medidas investigadas en los tres pilares de la sustentabilidad. Por último, no se constató diferencias en el desempeño financiero entre empresas de los dos grupos.

PALABRAS CLAVE: responsabilidad corporativa, turismo, Brasil. 


\section{INTRODUÇÃO}

No Brasil, a quantidade de empresas privadas que investem em ações de responsabilidade tem sido crescente (IPEA, 2006), assim como a preocupação acadêmica sobre o tema. A responsabilidade socioambiental corporativa (RSC) pode ser entendida como aquele conjunto de comportamentos voluntários das empresas que demonstram compromissos com questões sociais, ambientais e econômicas, ligadas ao tripé da sustentabilidade (Ashley, 2005; Bernardes, 2006; Bittencourt \& Carrieri, 2005; Bufoni, Muniz, \& Ferreira, 2009).

Quanto aos motivos para que as empresas adotem ações de responsabilidade, podem ser apontados diversos caminhos. Fica nítida a existência de divergências sobre o principal motivo de as empresas disponibilizarem tempo e dinheiro para projetos voluntários que denotam responsabilidade. A literatura identifica ao menos três com recorrência, sendo elas: estratégia, pressões externas ou valores internos (C. Ashley \& Haysom, 2006; Carrieri, Da Silva, \& Pimentel, 2009; Moretti \& Campanario, 2009).

A discussão dicotomizada entre a bondade ou o interesse também já foi explorada anteriormente. Nesta linha, um estudo brasileiro concluiu que as empresas não investem em ações de responsabilidade social motivadas apenas por uma ou outra razão (Peliano, 2001), mas sim que ambas estão presentes na decisão, revelando distintas motivações que levam as empresas a agirem de forma responsável. Já o estudo realizado pelo Ipea em 2006, especificamente, indicou que as convicções pessoais dos dirigentes das empresas, tanto filantrópicas quanto religiosas, tiveram destaque na entrada das empresas em ações responsáveis (IPEA, 2006 p. 26), quando comparadas com razões ligadas à imagem da empresa e outros aspectos.

Adensar o entendimento sobre estas motivações, especificamente no setor de turismo, é a proposta desta pesquisa. Afinal, ainda são poucos os estudos ligados à responsabilidade social e ambiental de empresas do setor de turismo no Brasil. Entre aqueles que se destacam no tema, encontra-se a pesquisa de Carvalho e Alberton (2007) acerca dos meios de hospedagem na estrada Real (MG). Neste trabalho, as autoras identificaram que a maioria das empresas entrevistadas (57\%) não praticava quaisquer ações de responsabilidade social corporativa (RSC). Assim, apesar de a pesquisa identificar as ações mais praticadas, ainda não possibilitou inferir as motivações de tais empresas para realizarem tais práticas.

Nestesentido,Souzae Costa(2016) propuseram uma aproximação ao tema ao investigar as razões que explicavam o comportamento responsável em pequenas operadoras de turismo por meio de um estudo de caso na Chapada dos Veadeiros (GO). Os autores indicaram uma multiplicidade de razões alegadas, sendo marcante a presença de aspectos chamados de não econômicos (valores pessoais, senso de comunidade, realização, entre outros), em contraposição aos chamados econômico-racionais e ligados ao desempenho da empresa. O trabalho aqui apresentado, por sua vez, propõe expandir este universo de empresas e ampliar a visão sobre a questão na realidade nacional e para outros segmentos de negócios vinculados ao turismo.

Internacionalmente, estudos como os de Font et al. (2014) e de Park e Kim (2014) contribuem para a reflexão aqui proposta. Ambos ressaltam a possibilidade de influência de características do local e do dono da empresa sobre a gestão, ou seja, indicam a existência de um conjunto de variáveis que, potencialmente, influenciam sobre as razões para adoção de práticas responsáveis no contexto do turismo (Garay \& Font, 2012). Há indícios em pesquisas realizadas na Europa de que existem múltiplos grupos de empresas diferenciados a partir de suas motivações para tomar atitudes responsáveis (Garay \& Font, 2012), ponto de partida para este estudo.

Assim, este trabalho se propõe a identificar as ações de responsabilidade mais recorrentes e as razões que as fundamentam na realidade do turismo brasileiro. Para alcançar este objetivo, foi construído um referencial teórico - apresentado na segunda parte do artigo - assentado em duas abordagens encontradas na literatura sobre os motivos para adotar ações responsáveis em empresas. Em seguida, a terceira parte do trabalho descreve a abordagem metodológica adotada para a pesquisa e, na quarta parte, são descritos e discutidos os resultados. Por fim, são expostas as considerações finais.

\section{OS MOTIVOS PARA ADOTAR AÇÕES RESPONSÁVEIS}

A revisão teórica foi organizada em duas vertentes contrastantes: (1) foco em aspectos do 
negócio, internos ou externos, relativos aos clientes, outros stakeholders e explicações econômicas e (2) foco em aspectos individuais, tais como escolhas ligadas ao estilo de vida e à noção da coisa certa a se fazer, à preocupação em proteger o meio ambiente e a sociedade. O primeiro foco revela uma perspectiva econômica-racional, enquanto o segundo não está limitado a ela e abarca questões não econômicas. Ao apresentar as duas visões, o referencial traz aportes para a reflexão acerca das RSC em relação ao porte das empresas e sua conexão com o setor de turismo.

\section{FOCO NOS NEGÓCIOS: BUSINESS CASE}

Ainda que a responsabilidade social corporativa (RSC) possa ser definida como a contribuição voluntária de uma empresa para a melhoria do meio ambiente, da sociedade e da economia (Blowfield \& Murray, 2008), a literatura tem enfatizado sua possível relação com a melhora dos resultados empresariais como uma das principais motivações para sua implementação, preponderando sobre elementos não econômicos. Assim, o chamado business case, ou foco no negócio, tem dominado grande parte da literatura sobre as motivações para introduzir a RSC (Carroll \& Shabana, 2010; Salzmann, Ionescu-Somers, \& Steger, 2005b), especialmente para o caso das grandes corporações e do setor industrial.

Alinhando-se a esta perspectiva, Carrieri et al. (2009) entendem que a empresa buscará ser social e ambientalmente responsável apenas quando isso traz retorno para ela, como de melhoria de imagem ou isenção de imposto de renda. Ou seja, os autores ligam o próprio conceito de RSC ao resultado do negócio. Para Carroll y Shabana (2010), uma forma simplista de entender o business case por parte dos gestores é como aquela visão que apenas leva em consideração uma implementação que proporcione um impacto direto e a curto prazo sobre o resultado financeiro da empresa, enquanto uma visão mais ampla levaria em conta todas as oportunidades que podem surgir da responsabilidade. Os autores destacam, especialmente, a aquisição de vantagens competitivas ligada à geração de recursos únicos, aspecto que tem recebido mais atenção e que está ligado à noção estratégica da visão baseada em recursos (RBV) (Barney, 1991; Inoue \& Lee, 2011).

A RBV sugere que a implementação das ações de responsabilidade podem ser motivadas pela possibilidade de originar recursos distintos e valiosos que poderão gerar uma vantagem competitiva para as empresas (Hart, 1995). E para que a vantagem possa ser sustentada ao longo do tempo, é necessário que estes recursos não sejam imitáveis sem grandes esforços por parte dos competidores e que realmente possam ser identificados como únicos no mercado (Inoue \& Lee, 2011). Ainda que as ações ligadas à sustentabilidade isoladas não possam ser classificadas como valiosas, raras, imperfeitamente imitáveis e com dificuldade de substituição (Barney, 1991), o conjunto das ações pode ser visto desta forma, já que muitas empresas não percebem a oportunidade para competitividade a partir delas (Bonilla-Priego, Najera, \& Font, 2011).

A busca de um bom desempenho financeiro como resultado da RSC domina maior parte da literatura atual e das políticas ligadas à RSC (Salzmann, Ionescu-Somers \& Steger, 2005a), colocando em segundo plano outras razões. A maior parte da literatura que relaciona lucros com RSC sugere que este relacionamento tem a forma de um U invertido. Ou seja, existe um nível ótimo de atividades de RSC que apoia o desempenho do negócio, e que agir além deste nível pode ser explicado apenas por um comportamento altruísta e não puramente comercial (Salzmann et al., 2005a). Em longo prazo, pode ser mais cauteloso aceitar que existe um relacionamento bidirecional no qual tanto RSC e desempenho financeiro apoiam-se mutuamente (Garay \& Font, 2012), porque a existência de lucro é tomada como prerrequisito para implementar ações voltadas para a sustentabilidade (Fassin, Van Rossem \& Buelens, 2011). Ao lucro soma-se também um boa governança corporativa, já que empresas bem gerenciadas e com alto desempenho deveriam ser mais capazes (ainda que não necessariamente mais propensas) a implementar ações voltadas para a sustentabilidade (Bonilla-Priego, et al., 2011).

Nos estudos revela-se controversa a relação entre a adoção da RSC e os resultados efetivos. Os trabalhos mais gerais a respeito não são conclusivos, já que revelam associações das mais diversas (positivas, negativas e neutras) entre estas variáveis. Todavia, estas pesquisas tendem a não orientar seu foco para a motivação que guia a implementação da RSC (Griffin \& Mahon, 1997).

A abordagem de RSC também tem sido concentrada na realidade de grandes empresas privadas (Farias \& Teixeira, 2002). As organizações grandes, especialmente aquelas dedicadas a setores como petróleo, mineração e celulose 
(Vinha, 2003), sofrem diversas pressões de seus investidores e outros atores para se dedicarem a ações responsáveis. As principais motivações para isso, conforme os autores, seria um potencial ganho de imagem, aliado a uma esperada responsividade às pressões externas, ambas entendidas como razões no campo racionaleconômico. Todavia, demonstra-se limitada atenção da literatura sobre empresas de pequeno porte, ainda que esta abordagem fosse importante, sobretudo, ao se refletir sobre o setor de turismo, que é um setor composto majoritariamente por pequenas empresas no Brasil (IBGE, 2007).

Detodaforma,tambémpodemexistirmotivadores econômicos para a implementação de RSC por parte das pequenas e médias empresas (PME). Ou seja, não se pode dizer que as empresas de pequeno porte não se motivam por questões econômicas quando da implementação de ações de responsabilidade. Ainda que se mencione habitualmente que os valores dos empresários são critérios básicos para introduzir ações responsáveis (Spence, Schmidpeter, \& Habisch, 2003b), encontram-se também entre estes valores o pragmatismo econômico e/ou a necessidade de manter certo posicionamento no mercado, bem como responder à pressão de certos stakeholders (Murillo \& Lozano, 2006). Para Murillo e Lozano (2006), ainda que a RSC, no caso das PME, não detenha um complexo sistema de gestão, pode constituir para estas empresas um sinal de identidade referente a sua excelência.

No setor de turismo, o motivo mais argumentado para implementar a RSC tem sido o de favorecer o desenvolvimento de recursos, alinhado com o objetivo clássico de maximizar os resultados criando vantagens competitivas (Knowles, Macmillan, Palmer, Grabowski \& Hashimoto, 1999; Stabler, 1997). Já existem diversos autores que indicam como positiva a relação entre responsabilidade e resultados financeiros no setor de turismo (Kang, Lee \& Huh, 2010; Lee \& Park, 2009; Molina-Azorín, Claver-Cortés, Pereira-Moliner \& Tarí, 2009; Rodriguez \& Del Mar Armas Cruz, 2007). Há estudos que também indicam que as empresas do setor turístico estão respondendo às novas expectativas da demanda por comportamentos mais responsáveis (Bremner, 2009). No entanto, a real demanda por ações de RSC por parte dos clientes, tanto no turismo quanto em outros setores, ainda não é pacificada (Lenziardi, Mayer \& Ferreira, 2010; Serpa \& Fourneau, 2007).

\section{FOCO NOS INDIVÍDUOS: ESTILO DE VIDA E VALORES PESSOAIS}

Em contraposição à argumentação da seção anterior, há abordagens que indicam a predominância de algumas razões mais individuais para que as empresas se comportem de forma responsável em virtude de uma combinação de considerações éticas, pessoais e econômicas por parte dos proprietários (Tzschentke, Kirk \& Lynch, 2004, 2008a). Essa visão abre para a consideração acerca dos portes das empresas envolvidas e tende a ressaltar as micro, pequenas e médias empresas.

Garay e Font (2013) indicaram evidências de que as empresas pequenas e médias do turismo estavam ligadas com questões de altruísmo baseadas em estudos realizados na China, no Chile e em países da Europa. Bernardes (2006), por sua vez, ao estudar micro e pequenas empresas situadas na capital do Brasil, revela que a motivação mais evidenciada pelos empresários é a ética pessoal, ou seu próprio código individual de valores, enquanto o retorno financeiro aparecia como a motivação de uma parcela muito pequena dos respondentes. Existem, portanto, indícios de que grande parte do comportamento observado em favor da RSC, especialmente quando se trata de empresas pequenas, é mais bem explicado pelos hábitos e estilos de vida dos proprietários/gerentes do que por ações conscientes ou em favor de ganhos para o negócio. Segundo a literatura, nas empresas de pequeno porte, os valores dos proprietários (morais, religiosos, pessoais, comunitários, etc.) são um fator chave na introdução da RSC (Jenkins, 2006; L. J. Spence, 2007).

Da combinação da importância dos valores com o caráter empreendedor, surge o conceito de Lifestyle Entreperneur (Shaw \& Williams, 2004). O sucesso para estes empresários se baseia em suas próprias circunstâncias e valores (Carlsen, Morrison \& Weber, 2008), que implicam uma gestão de seu negócio baseada em valores que, algumas vezes (mas não sempre), estão associados à responsabilidade (Ateljevic \& Doorne, 2000a).

Os motivos do proprietário para estabelecer e gerir o negócio tem se mostrado relevantes para a explicação do comportamento de empresários de MPE do turismo (P. Dewhurst \& Horobin, 1998; Hall \& Rusher, 2004; Horobin \& Long, 1996; Thomas, 1998). Ficam evidenciados que 
os objetivos e as ações das pequenas empresas são fortemente influenciados por fatores não econômicos, sobretudo pelo estilo de vida dos dirigentes (Dahlstrand \& Biel, 1997; Hall \& Rusher, 2004). Questões pessoais, como escolha por morar em determinado local pitoresco, alcançar uma vida mais calma e praticar hobbies, aparecem como fatores decisivos (Hall \& Rusher, 2004; Thomas, 1998, 2004). A ética pessoal e os comportamentos ligados ao estilo de vida são tão importantes para esse perfil como o fato de oferecer um serviço de alta qualidade e, por vezes, mais importante do que a maximização do lucro (Ateljevic \& Doorne, 2000a; Shaw \& Williams, 2004).

Sampaio et al. (2012b) ressaltaram que a visão de mundo influencia o engajamento ambiental e guia a seleção de quais medidas de responsabilidade adotar. Contudo, apesar de haver evidências da predominância de motivações não econômicas para tomada de decisões entre os empresários de PME de turismo, é importante fazer um contraponto: as prioridades de tais empresários não são necessariamente coletivas. Algumas delas tendem a ser centradas em si (em objetivos como independência, liberdade, criatividade), em seus clientes ou investidores (Dewhurst \& Horobin, 1998). Assim, pode-se perceber que motivações não econômicas não garantem, automaticamente, maior compromisso com condições mais favoráveis para a coletividade ou com aspectos comuns que têm ligação com a sustentabilidade.

Ainda que as razões principais possam não ser econômicas, saúde financeira é vista como um requisito para empreender ações de responsabilidade (Fassin et al., 2011). Todavia, na realidade de empresas pequenas, outras barreiras aparecem ao lado desta, tais como: a escassa formação dos proprietários/gerentes em tais aspectos (Roberts, Lawson \& Nicholls, 2006; Tilley, 2000), a crença de que a responsabilidade é cara e complexa (Dodds \& Holmes, 2010; Revell \& Blackburn, 2007) ou a percepção de deterem poder limitado em relação à dinâmica do setor (Lepoutre \& Heene, 2006; Zschiegner, 2011). Se não existirem outras motivações não econômicas, estes elementos podem provocar uma baixa implementação ou uma introdução muito básica de ações de responsabilidade, normalmente baseadas na expectativa de reduzir custos operacionais sob o discurso do "ecológico" (Font \& Harris, 2004).

\section{MÉTODOS}

O instrumento de coleta de dados foi originalmente desenvolvido em 2010 e testado na Catalunha, Espanha (Garay \& Font, 2012). Após passar por aprimoramentos em 2011 em aplicação no Chile, foi traduzido para o português a fim de ser empregado nesta pesquisa. As perguntas foram alteradas para se adaptar a diferenças nacionais, mantendo-se os constructos chaves. As questões, direcionadas a proprietários/gestores de empresas, abordavam aspectos relativos a: características empresariais; o desempenho dos negócios; os dados de origem do cliente e informações acerca do respondente. Solicitou-se que os entrevistados indicassem quais medidas ambientais, sociais e econômicas eram realizadas pela empresa, bem como as quatro principais razões para agir de forma responsável (dentre 16 opções) e as três principais barreiras para implementação de tais medidas (dentre 7 barreiras listadas). Em busca de atenuar o viés da desejabilidade social, optouse pelo anonimato nas respostas (Fuller \& Tian, 2006; Randall \& Fernandes, 1991).

A fim de identificar o universo da pesquisa, foi utilizado o CADASTUR, banco de dados oficial do Ministério do Turismo ${ }^{1}$. Foram consideradas, inicialmente, as 49.934 empresas que estavam na base e continham endereço eletrônico. Contudo, após reduzir duplicatas e eliminar endereços inválidos, o convite foi enviado por e-mail a 33.376 empresas. Foram obtidas 1.365 respostas ao questionário eletrônico após o prazo de duas semanas, totalizando uma taxa de resposta de $4 \%$.

$\mathrm{Na}$ análise dos dados foram empregadas técnicas estatísticas. Além da estatística descritiva, utilizou-se a Análise de Cluster K-means para agrupar os entrevistados com base nas motivações para introduzir medidas de sustentabilidade. K-means é um método de análise de cluster, que visa dividir n observações em k grupos, em que cada observação pertence ao cluster com a média mais próxima. A amostra original (sem ordenação de variáveis) foi utilizada para testar diferentes clusters no SPSS $®$. As três primeiras rodadas de testes excluíram cinco variáveis que não apresentaram diferenças significativas. O quarto teste deu um resultado consistente, no qual todas as variáveis restantes (motivos para implementar ações

1 Os autores gostariam de agradecer ao Ministério do Turismo por sua colaboração. 
responsáveis) mostraram diferenças significativas. Quando testado por meio de uma análise de variância, todas as motivações tinham um valor de F com significância inferior a 0,05. Este teste apresentou dois grupos que eram consistentes com a literatura e que ofereceram valor interpretativo. Então, as diferenças foram analisadas por meio de tabulações cruzadas e testes qui-quadrado para as variáveis categóricas. Para as variáveis intervalares, empregou-se a análise de médias juntamente com análise de variância ANOVA.

A maioria dos respondentes foi composta por homens (61,2\%). Dentro da amostra, houve um elevado número de proprietários $(75,8 \%)$ e de gerentes (24,2\%). Quanto ao perfil das empresas, maior parte era familiar (72,7\%), micro ou pequenas (57,3\% com menos de 5 funcionários e $88 \%$ com 10 ou menos), relativamente jovens $(67,9 \%$ com menos de 10 anos e $43,8 \%$ com até 5 anos) e quase a totalidade não fazia parte de qualquer cadeia ou franquia (92,8\%). Assim, ainda que a pesquisa não tenha focado em pequenas empresas, é natural esta composição das respostas, frente ao fato de que $97,7 \%$ das empresas de turismo do Brasil são pequenas, com até 19 empregados (IBGE, 2007).

A distribuição das empresas respondentes por tipo de atividade foi variada. Dentre os respondentes, 30,5\% eram agências de viagens, $22 \%$ eram meios de hospedagem, 11,9\% eram empresas de transporte turístico. Com participação abaixo de $10 \%$, obtiveram-se $9,0 \%$ de restaurantes e similares, $8,9 \%$ de agências de receptivo e guias, $3,5 \%$ de empresas de aluguel de carros e $2,4 \%$ de operadores de turismo de aventura. Os clientes dessas empresas eram predominantemente regionais $(60,6 \%)$, seguidos por turistas nacionais de outros estados (33,2\%). Por outro lado, existe apenas uma baixa percentagem de clientes internacionais (6,2\%). A impressão geral dos entrevistados sobre a situação financeira da empresa que estavam gerenciando, bem como suas perspectivas futuras, indicou uma visão mais favorável do que desfavorável. De modo mais detalhado, 60,6\% disseram que a situação financeira de sua empresa é regular, enquanto e $22,1 \%$ afirmaram que é boa, e apenas $17,4 \%$ entenderam que é ruim. Finalmente, a maioria das empresas $(87,1 \%)$ não apresentava quaisquer certificados de sustentabilidade, mas o número de firmas que os têm $(12,9 \%)$ parece significante, considerando experiências semelhantes em outros países ou regiões.

\section{APRESENTAÇÃO E DISCUSSÃO DE RESULTADOS}

Os resultados foram organizados em duas partes. A primeira descreve as práticas encontradas em suas dimensões sociais, ambientais e econômicas, bem como discute a visão comparativa das empresas nos quesitos investigados. A segunda parte é dedicada a apresentar as razões alegadas para a implementação de tais práticas, bem como analisar a formação de clusters a partir dos resultados.

\section{PRÁTICAS DE RESPONSABILIDADE E VISÃO COMPARATIVA NAS TRÊS ESFERAS DA SUSTENTABILIDADE}

Em relação às práticas responsáveis que as empresas investigadas estão introduzindo (Tabela 1), dentro de cada dimensão do tripé da sustentabilidade, pode-se ver que os aspectos ambientais foram destacados como os mais adotados. Em contrapartida, os sociais foram os menos implementados.

No que diz respeito a medidas ambientais, dois terços da amostra de empresas que implementam medidas para economizar energia ou água (ecosavings). Neste contexto, a economia de energia é a medida mais referida em toda a amostra, com uma parcela de $82,7 \%$. Outra ação notável é aquela relacionada com o fato de encorajar os clientes a serem ambientalmente responsáveis, com o percentual de 57,7\%. Esta ação também estabelece uma relação com as formas de comunicar ações responsáveis e o impacto que isso pode ter sobre os clientes.

Deve-se salientar que outras medidas - tais como a escolha de fornecedores que demonstram responsabilidade ambiental, o uso de produtos biodegradáveis, a reciclagem de resíduos sólidos e a execução de ações que contribuem para a conservação da biodiversidade local - estão localizadas em percentuais elevados da amostra, entre $35 \%$ e $45 \%$. Isso indica, no que tange à dimensão ambiental da sustentabilidade, que essas empresas estão avançando na incorporação de algumas medidas que requerem uma gestão mais complexa e mudanças organizacionais mais expressivas. Quanto à pequena percentagem que demonstra o uso de fontes alternativas de energia, uma das possíveis explicações é o custo fixo elevado para instalação de equipamentos e tecnologias, especialmente na realidade das micro e pequenas empresas, maioria dos respondentes da pesquisa. 
Tabela 1 - Práticas de responsabilidade

\begin{tabular}{|c|c|c|}
\hline Ambiental & $\mathrm{n}$ & $\%$ \\
\hline Economiza energia & 1112 & $82,7 \%$ \\
\hline Poupa água & 1038 & $77,2 \%$ \\
\hline Incentiva seus clientes a serem ambientalmente responsáveis & 775 & $57,7 \%$ \\
\hline Escolhe fornecedores que demonstram responsabilidade ambiental & 610 & $45,4 \%$ \\
\hline Utiliza produtos biodegradáveis & 569 & $42,3 \%$ \\
\hline Recicla resíduos sólidos & 520 & $38,7 \%$ \\
\hline Realiza ações que contribuem para a conservação da biodiversidade local & 476 & $35,4 \%$ \\
\hline Recicla resíduos líquidos & 248 & $18,5 \%$ \\
\hline Utiliza energias alternativas (solar, eólica, biomassa) & 184 & $13,7 \%$ \\
\hline \multicolumn{3}{|l|}{ Social } \\
\hline Difunde e/ou incentiva a preservação da cultura e do patrimônio locais & 738 & $55,1 \%$ \\
\hline Promove a igualdade de gênero em suas atividades & 686 & $51,2 \%$ \\
\hline $\begin{array}{l}\text { Implementa medidas para equilibrar o trabalho e a vida pessoal dos seus } \\
\text { trabalhadores }\end{array}$ & 654 & $48,8 \%$ \\
\hline Escolhe fornecedores que demonstram responsabilidade social & 612 & $45,7 \%$ \\
\hline Incentiva seus clientes a contribuírem para iniciativas sociais & 546 & $40,8 \%$ \\
\hline $\begin{array}{l}\text { Difunde e/ou incentiva o desenvolvimento da comunidade local, exemplo: } \\
\text { voluntariado }\end{array}$ & 428 & $32,0 \%$ \\
\hline Dispõe de instalações para pessoas com algum tipo de dificuldade de locomoção & 424 & $31,7 \%$ \\
\hline Emprega pessoas com deficiências & 168 & $12,5 \%$ \\
\hline \multicolumn{3}{|l|}{ Econômica } \\
\hline Preferencialmente contrata pessoas que vivem onde a empresa está localizada & 1011 & $75,2 \%$ \\
\hline Dá prioridade para a compra de produtos ou serviços de fontes locais & 949 & $70,6 \%$ \\
\hline Incentiva seus clientes para o consumo de produtos locais & 813 & $60,5 \%$ \\
\hline Oferece benefícios adicionais (monetários e não monetários) para seus funcionários & 753 & $56,0 \%$ \\
\hline Possui programas de treinamento para os funcionários de sua empresa & 567 & $42,2 \%$ \\
\hline
\end{tabular}
Fonte: dados da pesquisa

Nota: "n" é o número de entrevistados e "\%" o percentual válido sobre a amostra (excluindo as respostas inválidas). Fonte: Dados da pesquisa

Tabela 2 - Visão comparativa das percepções das empresas sobre sua adoção de medidas responsáveis nas três esferas

\begin{tabular}{|c|c|c|c|}
\hline & Ambiental & Social & Econômica \\
\hline Na média & 48,1 & 47 & 50,1 \\
\hline Acima da média & 15,6 & 11,8 & 17,5 \\
\hline Abaixo da média & 11,9 & 16,5 & 10,9 \\
\hline Não sabe & 24,4 & 24,7 & 21,4 \\
\hline
\end{tabular}

Fonte: Dados da pesquisa

Quando questionados sobre sua percepção acerca da incorporação dessas medidas por seus empreendimentos, os dados deixam entrever (Tabela 2) que quase metade dos respondentes pensa que suas empresas não se diferenciam de outras neste quesito, estando na média do setor. Um número semelhante delas indica estar acima ou abaixo desta média. Entre estes dados, uma das apurações mais significativas reside no fato de um quarto dos respondentes não saber se posicionar 
em relação a outras empresas quando o assunto é adoção de medidas de responsabilidade ambiental, o que enfraquece a visão da responsabilidade adotada como diferencial competitivo, aspecto que tende a ser ressaltado pela literatura.

Dentre as práticas na esfera social da sustentabilidade, duas das medidas investigadas apresentam taxas de adesão acima de 50\%, especificamente ligadas à preservação da cultura e do patrimônio locais, bem como à promoção da igualdade de gênero em suas atividades. Todavia, outras práticas, como a implementação de ações para equilibrar trabalho e vida pessoal de seus funcionários, escolha de fornecedores que demonstram responsabilidade social, incentivo a seus clientes para contribuir com iniciativas sociais, difusão e incentivo do desenvolvimento da comunidade local e oferta de instalações para as pessoas com algum tipo de mobilidade reduzida, são implementadas, entre $30 \%$ e $50 \%$ da amostra. Somente quanto a empregar pessoas com deficiência há uma incidência bastante menor do que as demais medidas, com apenas $12,5 \%$ dos respondentes demonstrando realizar esta ação.

Quanto às percepções acerca da implementação destas medidas sociais, evidenciam-se patamares semelhantes àqueles ligados à esfera ambiental (Tabela 2). A partir disso, é possível inferir que os respondentes tendem a perceber que estão mais avançados na questão ambiental do que no aspecto social em seus empreendimentos.

Em terceiro lugar, no que diz respeito às medidas econômicas, pode-se constatar que, em geral, os percentuais são altos. Destaca-se com o mais alto escore a prática de contratação de pessoas que vivem onde a empresa está localizada, correspondendo a pouco mais de dois terços da amostra. Nesta esfera, praticamente todas as medidas situam-se no terceiro quartil da amostra, o que demonstra uma maior homogeneidade entre as empresas neste quesito e progresso nesta dimensão do comportamento responsável. Apenas uma medida, referente a ter programas de treinamento para os funcionários da própria empresa, está sozinha e com um peso ligeiramente inferior à metade das empresas (42,2\%). Comparando com as demais dimensões (Tabela 2), aparentemente, a dimensão econômica da sustentabilidade é aquela que empresas percebem estarem mais avançadas. Em contrapartida, a dimensão social é que aparenta estar menos desenvolvida por elas.

\section{MOTIVOS PARA SER RESPONSÁVEL: RAZÕES E AGREGAÇÃO EM CLUSTERS}

A Tabela 3 apresenta os resultados sobre a razão pela qual as ações de responsabilidade foram introduzidas nas empresas. As principais razões são socioambientais, tais como proteger o meio ambiente e melhorar a sociedade, com taxas superiores a $50 \%$ (o primeiro, com cerca de $65 \%$ ). Por outro lado, é interessante notar como estas são seguidas pela tradicional visão do negócio, como a redução de custos, o atendimento de exigências legais e para alcançar benefícios de marketing e imagem da empresa, com pesos entre $30 \%$ e $50 \%$. Encontra-se em terceiro lugar outro argumento chave, a manutenção de um estilo de vida, com $28 \%$ das respostas, mas longe dos principais motivos relatados e especialmente longe do que foi encontrado em outros estudos, tais como Garay e Font (2012). Por fim, com peso inferior a 26\%, encontram-se outras razões, tais como alguns aspectos relacionados à competitividade da economia e outros, com um peso marginal, outros relacionados à manutenção de uma certa legitimidade perante alguns stakeholders (operadores turísticos, cadeia ou grupo empresarial).

Tabela 3 - Motivos para ser responsável

\begin{tabular}{|c|c|c|}
\hline Para proteger o meio ambiente & $\mathbf{n}$ & \% \\
\hline Para melhorar a nossa sociedade & 713 & $52,80 \%$ \\
\hline Para redução de custos & 641 & $47,50 \%$ \\
\hline Para atender aos requisitos legais & 414 & $30,70 \%$ \\
\hline $\begin{array}{c}\text { Para benefícios de marketing e } \\
\text { imagem }\end{array}$ & 413 & $30,60 \%$ \\
\hline $\begin{array}{c}\text { Questões pessoais/meu estilo de } \\
\text { vida }\end{array}$ & 381 & $28,20 \%$ \\
\hline $\begin{array}{c}\text { Reduzir os riscos da empresa } \\
\text { por conta da degradação do meio } \\
\text { ambiente a longo prazo }\end{array}$ & 362 & 26,80 \\
\hline $\begin{array}{c}\text { Para ser mais competitiva no } \\
\text { mercado global }\end{array}$ & 358 & $26,50 \%$ \\
\hline $\begin{array}{c}\text { Melhorar o monitoramento/ } \\
\text { controle do funcionamento da } \\
\text { empresa }\end{array}$ & 285 & $21,10 \%$ \\
\hline $\begin{array}{c}\text { Acesso a novas informações/ } \\
\text { conselho/redes }\end{array}$ & 232 & $17,20 \%$ \\
\hline $\begin{array}{c}\text { Em resposta à demanda dos } \\
\text { clientes }\end{array}$ & 225 & $16,70 \%$ \\
\hline
\end{tabular}




\begin{tabular}{|c|c|c|}
\hline $\begin{array}{c}\text { Aumentar o faturamento da } \\
\text { empresa por meio do alcance dos } \\
\text { clientes que gastam mais }\end{array}$ & 140 & 10,40 \\
\hline $\begin{array}{c}\text { Para atender as exigências de um } \\
\text { operador turístico }\end{array}$ & 140 & $10,40 \%$ \\
\hline Para obter subsídios & 110 & $8,10 \%$ \\
\hline $\begin{array}{c}\text { Para atender às exigências de nossa } \\
\text { cadeia/grupo }\end{array}$ & 89 & $6,60 \%$ \\
\hline
\end{tabular}

Fonte: Dados da pesquisa

$\mathrm{Na}$ análise de clusters com o uso do método $\mathrm{K}$-means, identificaram-se diferenças significativas em todas as razões entre dois aglomerados, os quais, neste caso, estão bem definidos ao redor de características próprias. A Tabela 4 explicita como esses dois grupos estão relacionados com as razões para adotar práticas responsáveis. Podese observar que existem dois perfis distintos: o primeiro grupo inclui empresas que alegam como principais razões para adotarem ações responsáveis as questões pessoais, os valores (ligados à proteção ambiental e à melhoria social) e o estilo de vida. Este grupo é mais numeroso dentre as empresas em questão, já que engloba 58,9\% delas.

Por outro lado, o segundo grupo se preocupa especialmente com razões relacionadas aos negócios e à conformidade legal, tais como redução de custos, atendimento aos requisitos regulatórios, à competitividade ou à comercialização. As motivações ligadas com os stakeholders (clientes, cadeia ou grupo) são também significativamente mais elevadas para este grupo em relação ao primeiro, ainda que tenha um peso baixo para ambos. Na mesma Tabela 4, esses motivos estão ordenados de acordo com seu peso para demonstrar as diferenças significativas entre os dois grupos.

Tabela 4 - Clusters dos motivos para implementar práticas de responsabilidade e resultados

\begin{tabular}{|c|c|c|c|c|c|c|c|c|c|c|c|c|c|c|c|c|}
\hline & $\omega$ & 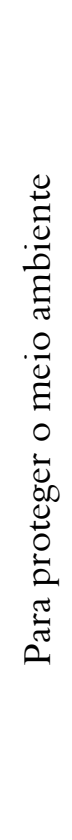 & 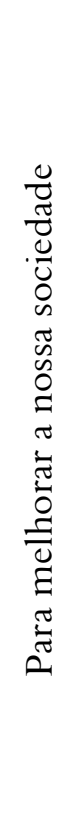 & 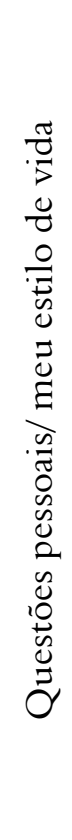 & 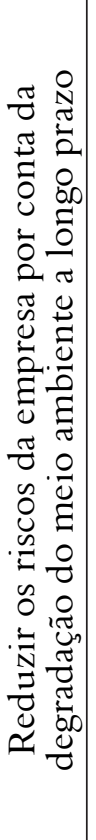 & 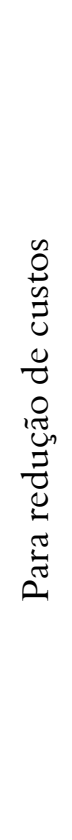 & 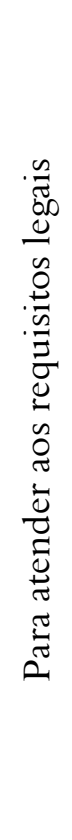 & 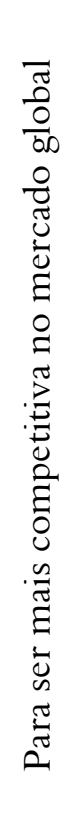 & 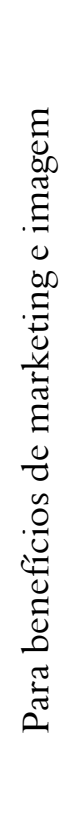 & 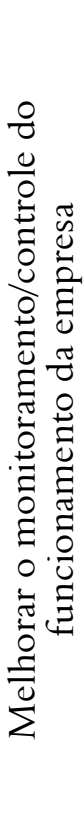 & 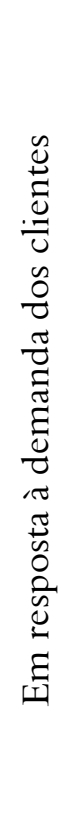 & 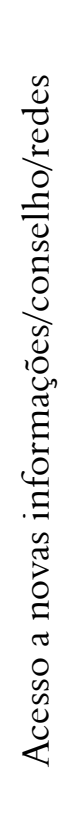 & 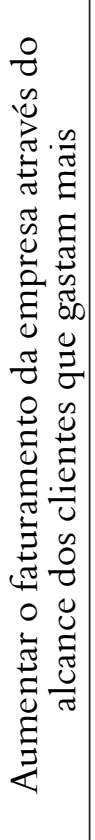 & 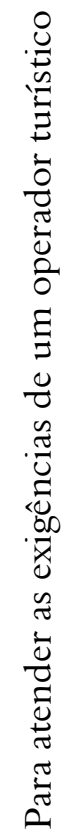 & 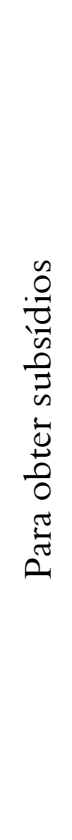 & 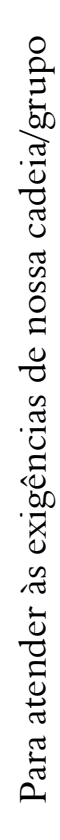 \\
\hline \multirow{2}{*}{$\begin{array}{c}\text { Foco no } \\
\text { indiví- } \\
\text { duo } \\
(\mathrm{n}= \\
795)\end{array}$} & $\mathrm{M}$ & 0,87 & 0,76 & 0,43 & 0,41 & 0,39 & 0,22 & 0,16 & 0,26 & 0,12 & 0,08 & 0,11 & 0,04 & 0,06 & 0,03 & 0,04 \\
\hline & S & 0,33 & 0,43 & 0,50 & 0,49 & 0,49 & 0,42 & 0,37 & 0,44 & 0,33 & 0,27 & 0,32 & 0,18 & 0,24 & 0,18 & 0,20 \\
\hline \multirow{2}{*}{$\begin{array}{c}\text { Foco no } \\
\text { negócio } \\
(\mathrm{n}= \\
555)\end{array}$} & $\mathrm{M}$ & 0,32 & 0,19 & 0,07 & 0,07 & $\mathbf{0 , 5 9}$ & 0,43 & 0,41 & 0,37 & 0,34 & 0,29 & 0,26 & 0,20 & 0,17 & 0,15 & 0,10 \\
\hline & S & 0,47 & 0,40 & 0,26 & 0,26 & 0,49 & 0,50 & 0,49 & 0,48 & 0,47 & 0,45 & 0,44 & 0,40 & 0,37 & 0,36 & 0,30 \\
\hline \multirow{2}{*}{$\begin{array}{l}\text { Total } \\
\mathrm{N}= \\
1350\end{array}$} & M & 0,65 & 0,53 & 0,28 & 0,27 & 0,47 & 0,31 & 0,27 & 0,31 & 0,21 & 0,17 & 0,17 & 0,10 & 0,10 & 0,08 & 0,07 \\
\hline & S & 0,48 & 0,50 & 0,45 & 0,44 & 0,50 & 0,46 & 0,44 & 0,46 & 0,41 & 0,37 & 0,38 & 0,31 & 0,31 & 0,27 & 0,25 \\
\hline
\end{tabular}

Fonte: Dados da pesquisa

Nota: $M=$ Média, como percentual de empresas ( 0 a 1 ) que apresentam esta motivação em cada cluster. $S=$ Desvio padrão. Fonte: Dados da pesquisa 
Considerando as diferenças significativas entre estes dois grupos, há resultados importantes em relação a algumas variáveis. Por exemplo, em termos de gênero, as empresas com foco no indivíduo têm proporcionalmente mais mulheres como proprietários ou em cargos de direção das empresas, enquanto no grupo de negócios há uma proporção maior de homens. Os clusters também revelam diferenças nos tipos de atividades predominante em cada grupo. No grupo de foco no indivíduo, há uma maior proporção de empresas alojamento e restaurantes, enquanto no grupo de foco nos negócios existe, proporcionalmente, um maior número de agências de viagens e empresas de transporte. Há também diferenças significantes na origem de clientes, com uma maior proporção relativa de clientes regionais e internacionais no grupo que tem foco no indivíduo.

Os dados não revelam diferenças significantes entre os grupos no que tange à empresa ser certificada ou não, a pertencer a uma cadeia ou grupo, ao tamanho, à idade da empresa ou ao fato de que é familiar ou não. Finalmente, um dos resultados mais interessantes a este respeito é o fato de que não há uma relação significante entre pertencer a um dos dois grupos identificados e o fato de ter uma melhor situação financeira ou uma melhora recente nos resultados financeiros da empresa. Assim, o resultado financeiro não parece variar de acordo com a motivação para implementar as ações de responsabilidade.

$\mathrm{Na}$ Tabela 5, pode-se verificar as diferenças entre os dois grupos em relação à introdução de medidas de responsabilidade. Constata-se uma diferença significativa e positiva na execução da maior parte das medidas entre os dois grupos. O grupo que tem motivações centradas nos indivíduos evidencia uma tendência a implementar as medidas investigadas em um percentual mais expressivo, em praticamente nos três pilares da sustentabilidade.

Tabela 5 - Práticas de responsabilidade e resultados gerais

\begin{tabular}{|c|c|c|c|c|c|}
\hline & $x^{2}$ & $\mathrm{p}$ & Indivíduos & Negócios & Amostra \\
\hline \multicolumn{6}{|l|}{ Ambiental } \\
\hline Economiza energia & 3.04 & 0.05 & 84.2 & 80.6 & 82.7 \\
\hline Poupa água & 0.11 & 0.39 & 77.6 & 76.8 & 77.2 \\
\hline $\begin{array}{c}\text { Incentiva seus clientes a serem ambientalmente } \\
\text { responsáveis }\end{array}$ & 37.05 & 0.00 & 64.6 & 47.7 & 57.7 \\
\hline $\begin{array}{l}\text { Escolhe fornecedores que demonstram } \\
\text { responsabilidade ambiental }\end{array}$ & 2.12 & 0.08 & 47.0 & 43.0 & 45.4 \\
\hline Utiliza produtos biodegradáveis & 10.07 & 0.00 & 45.9 & 37.2 & 42.3 \\
\hline Recicla resíduos sólidos & 13.43 & 0.00 & 42.7 & 32.8 & 38.7 \\
\hline $\begin{array}{l}\text { Realiza ações que contribuem para a conservação da } \\
\text { biodiversidade local }\end{array}$ & 11.42 & 0.00 & 39.1 & 30.1 & 35.4 \\
\hline Recicla resíduos líquidos & 0.91 & 0.18 & 19.3 & 17.2 & 18.5 \\
\hline Utiliza energias alternativas (solar, eólica, biomassa) & 10.87 & 0.00 & 16.3 & 10.0 & 13.7 \\
\hline \multicolumn{6}{|l|}{ Social } \\
\hline $\begin{array}{c}\text { Difunde e/ou incentiva a conservação da cultura e } \\
\text { do patrimônio locais }\end{array}$ & 10.93 & 0.00 & 58.9 & 49.7 & 55.1 \\
\hline Promove a igualdade de gênero em suas atividades & 18.09 & 0.00 & 56.1 & 44.3 & 51.2 \\
\hline $\begin{array}{l}\text { Implementa medidas para equilibrar o trabalho e a } \\
\text { vida pessoal dos seus trabalhadores }\end{array}$ & 7.20 & 0.00 & 51.9 & 44.4 & 48.8 \\
\hline $\begin{array}{l}\text { Escolhe fornecedores que demonstram } \\
\text { responsabilidade social }\end{array}$ & 2.77 & 0.05 & 47.6 & 43.0 & 45.7 \\
\hline $\begin{array}{c}\text { Incentiva seus clientes a contribuírem para } \\
\text { iniciativas sociais }\end{array}$ & 9.93 & 0.00 & 44.3 & 35.7 & 40.8 \\
\hline $\begin{array}{l}\text { Difunde e/ou incentiva o desenvolvimento da } \\
\text { comunidade local }\end{array}$ & 9.96 & 0.00 & 35.3 & 27.1 & 32.0 \\
\hline
\end{tabular}




\begin{tabular}{|c|c|c|c|c|c|}
\hline $\begin{array}{l}\text { Dispõe de instalações para pessoas com algum tipo } \\
\text { de dificuldade de locomoção }\end{array}$ & 5.07 & 0.01 & 34.1 & 28.2 & 31.7 \\
\hline Emprega pessoas com deficiências & 0.67 & 0.23 & 13.2 & 11.7 & 12.5 \\
\hline \multicolumn{6}{|l|}{ Econômica } \\
\hline $\begin{array}{l}\text { Preferencialmente contrata pessoas que vivem onde } \\
\text { a empresa está localizada }\end{array}$ & 0.64 & 0.23 & 76.0 & 74.1 & 75.2 \\
\hline $\begin{array}{l}\text { Dá prioridade para a compra de produtos ou } \\
\text { serviços de fontes locais }\end{array}$ & 12.26 & 0.00 & 74.2 & 65.4 & 70.6 \\
\hline $\begin{array}{l}\text { Incentiva seus clientes para o consumo de produtos } \\
\text { locais }\end{array}$ & 15.68 & 0.00 & 64.9 & 54.2 & 60.5 \\
\hline $\begin{array}{l}\text { Oferece benefícios adicionais (monetários e não } \\
\text { monetários) para seus funcionários }\end{array}$ & 1.58 & 0.11 & 57.4 & 54.0 & 56.0 \\
\hline $\begin{array}{l}\text { Há programas de treinamento para os funcionários } \\
\text { de sua empresa }\end{array}$ & 5.49 & 0.01 & 44.8 & 38.4 & 42.2 \\
\hline
\end{tabular}

Nota: Nas colunas Indivíduo, Negócio e Amostra é apresentado o percentual de respondentes em um perfil selecionando essa resposta. Células em negrito mostram diferenças significativas entre os clusters. Em todos os casos nenhuma célula $(0,0 \%)$ teve o valor esperado menor do que $5 \cdot \chi^{2}=$ valor do qui-quadrado, $\mathrm{p}=$ significância.

Este estudo confirma, portanto, a literatura que apresenta os motivos para realizar ações de responsabilidade como múltiplos. Não existe homogeneidade, mas sim grupos que se diferenciam por conta de suas razões para aderir a tais práticas. Foram identificados os tipos de ações das quais cada grupo participa e como isso se relaciona com características da empresa, a fim de apresentar uma representação mais complexa da realidade.

No caso das empresas de turismo brasileiras, as razões para elas serem responsáveis se diferenciam, mas não totalmente, dos três perfis encontrados na literatura (Dewhurst \& Thomas, 2003; Spence, Schmidpeter, \& Habisch, 2003a): um ligado aos benefícios esperados para o negócio, outro que orbitava ao redor da noção de devolver algo para a sociedade e, por fim, outro ligado a questões pessoais e estilo de vida. Neste estudo, encontraram-se apenas dois dos perfis esperados, havendo a combinação dos dois últimos em um mesmo grupo que agregou valores, sejam coletivos ou individuais, e estilo de vida. Os dois grupos não puderam ser associados com os respectivos portes das empresas, como poderia ser esperado a partir da literatura, o que talvez seja explicado pela prevalência de empresas pequenas na composição dos respondentes da pesquisa.

No estudo aqui empreendido, a motivação ligada à redução de custo e aos esforços para atingir competitividade foi explicada pela teoria baseada em recursos (RBV), que entendem as empresas como organizações racionais e perseguidoras de resultados econômicos. Foram encontrados cerca de $40 \%$ dos respondentes dentro deste grupo.

Por outro lado, mais de $58 \%$ dos respondentes fizeram parte do grupo de foco no indivíduo, ou seja, ligado a seus valores e estilo de vida. Estes são chamados por Dewhurst e Thomas (2003) de "atores comprometidos". Os autores afirmam que grande parte das ações de responsabilidade estão implícitas nas rotinas destes empresários, corroborandoSampaioetal. (2012). Para este grupo, realizar ações em favor da sustentabilidade não tem relação direta com desempenho do negócio, mas sim com uma satisfação com o resultado da empresa como parte de seus objetivos pessoais, como é visto em empresas altamente influenciadas pelo estilo de vida de seus proprietários e gerentes. Aqui também se encontram empresários muito confiantes na qualidade de seus serviços, avessos à comunicação de ações de responsabilidade e marketing, especialmente se isso prejudica sua integridade (Ateljevic \& Doorne, 2000b). Isso evidencia, portanto, que hábitos e estilo de vida podem ajudar a explicar a prática de ações responsáveis empresariais. Essa compreensão poderia ter implicações na concepção de programas de incentivo à prática da sustentabilidade no universo empresarial, bem como poderia ampliar a possibilidade de comunicação entre aqueles que buscam promover tais mudanças de atuação e as empresas. 


\section{CONSIDERAÇÕES FINAIS}

O estudo teve como objetivo identificar o comportamento responsável de empresas de turismo no Brasil, buscando compreender os motivos pelos quais implementam tais ações. Assim, seus resultados contribuem para compreender as razões, as práticas e os resultados ligados à gestão da sustentabilidade e da responsabilidade em empresas de turismo, especialmente de pequeno porte, e oferecer mais dados quantitativos para aplicação e refinamento dos perfis identificados por Dewhurst e Thomas (2003).

O referencial teórico apoiou o reconhecimento de dois grupos: um com foco nos negócios, ou em aspectos racionais-econômicos, e outro focado nos indivíduos, ou seja, ligado aos valores e ao estilo de vida do proprietário/gestor do empreendimento. Os dados da pesquisa, tomando como base a composição da amostra de respondentes, revelaram aspectos interessantes acerca de empresas familiares, independes e de micro ou pequeno porte do turismo brasileiro. Afinal, este foi o perfil predominante na pesquisa e que baliza a interpretação dos dados.

Entre as empresas de turismo do Brasil que responderam à pesquisa, foram identificadas que as medidas ambientais mais frequentemente implementadas são, principalmente, ligadas à economia de energia ou água, ou seja, o que se chama de ecosavings. Todavia, a representatividade de outras práticas ambientais mais complexas demonstra progressos.

Dentre as práticas sociais consideradas, destacam-se ações de preservação da cultura e do patrimônio locais, bem como a promoção da igualdade de gênero. Em contraposição, existe baixa representatividade no quesito de inclusão de pessoas com deficiência no ambiente de trabalho das organizações.

$\mathrm{Na}$ dimensão econômica, em geral, há evidência de ações em altas proporções, que tendem a somar metade ou mais das empresas. A que mais se destaca tem uma interface social, já que se refere à contratação de pessoas moradoras do local de instalação da empresa, e a que é menos recorrente diz respeito ao investimento $\mathrm{em}$ treinamento para seus empregados.

Comparando ações ligadas às três dimensões da sustentabilidade, aparentemente, a dimensão ambiental é a que apresenta mais altos percentuais de ações realizadas, quando consideradas as ações mais implementadas pelos respondentes. Em contrapartida, a dimensão social é que aparenta estar menos desenvolvida por elas. Então, quando manifestam sua visão comparada com outras empresas do setor do turismo no Brasil, ficou patente a alta incidência de respostas que demonstram não saber situar suas ações em relação a outras empresas, o que fragiliza a expectativa das ações de responsabilidade voltadas para gerar vantagens competitivas.

Quanto às razões para adotar práticas de responsabilidade, com o emprego da análise de clusters, foi possível identificar os dois aglomerados evidenciados na literatura: o grupo com foco nos indivíduos e o grupo com foco nos negócios, quando se trata da razão prevalecente para implementar ações de responsabilidade social, ambiental e econômica nas empresas de turismo. O primeiro é construído ao redor de empresas que mencionam como principais motivos as questões pessoais, os valores e o estilo de vida, sendo o mais proeminente de acordo com os resultados da pesquisa. Já o segundo grupo tende a ter razões vinculadas aos negócios, tais como redução de custos, atendimento aos requisitos regulatórios, entre outros.

A evidência de dois grupos permite inferir que existem diversos motivos para adotar medidas responsáveis quando se trata de empresas de turismo. As empresas respondentes refutam tal explicação como uma questão puramente comercial. Aparentemente, os instrumentos de estímulos a tais comportamentos precisariam carregar esta compreensão acerca dos indivíduos e dos seus conjuntos de valores pessoais (Sampaio, Thomas \& Font, 2012; Tzschentke, Kirk \& Lynch, 2008b; Vernon, Essex, Pinder \& Curry, 2003; Zschiegner, 2011). Quando se procura relacionar o desempenho financeiro percebido pelas empresas com os motivos anteriores, não há evidência de que tenham correlação. Ou seja, ainda que o foco seja no negócio, isso não garante um resultado superior para a empresa.

Por fim, ainda que os dados não tenham revelado uma relação significativa dos grupos em relação ao porte das empresas, o que pode ser explicado pela reduzida presença de empresas grandes na amostra, o estudo apresenta indícios que permitem conhecer melhor a realidade das ações de responsabilidade por parte das MPE do turismo brasileiras, que também poderiam ser mais exploradas futuramente. 
Como limitação deste estudo tem-se o viés, próprio da pesquisa on-line, acerca da autosseleção dos respondentes. Para aprofundamentos futuros, sugere-se um estudo mais detalhado do papel do altruísmo e da relação dele com os valores do indivíduo, tendo em vista refinar a análise. Ademais, dados qualitativos seriam importantes para diminuir a lacuna entre as respostas fornecidas e o comportamento real, bem como para chegar a interpretações detalhadas acerca dos motivos para adotar comportamentos mais sustentáveis, especialmente entre empresas de micro e pequeno porte.

\section{REFERÊNCIAS}

Ashley, A. P. (2005). Ética e Responsabilidade Social nos Negócios. São Paulo: Saraiva.

Ashley, C., \& Haysom, G. (2006). From philanthropy to a different way of doing business: strategies and challenges in integrating pro-poor approaches into tourism business. Development Southern Africa, 23(2), 265-280.

Ateljevic, I., \& Doorne, S. (2000a). Staying within the fence: lifestyle entrepreneurship in tourism. Journal of Sustainable Tourism, 8(5), 378-392.

Ateljevic, J. (2007). Small tourism firms and management practices in New Zealand: The Centre Stage Macro Region. Tourism Management, 28(1), 307-316.

Ateljevic, J., \& Doorne, S. (2000b). Staying within the fence: lifestyle entrepreneurship in tourism. Journal of Sustainable Tourism, 8(5), 378-392.

Barney, J. (1991). Firm Resources and Sustained Competitive Advantage. Journal of Management, 17(1), 99-120.

Bernardes, B. Q. T. (2006). Responsabilidade socioambiental das micro e pequenas empresas do DF. - Mestrado em Desenvolvimento Sustentável, Universidade de Brasília, Brasília.

Bittencourt , E., \& Carrieri, A. D. P. (2005). Responsabilidade Social: Ideologia, Poder e Discurso na Lógica Empresarial. RAE, 45.

Blowfield, M., \& Murray, A. (2008). Corporate responsibility: a critical introduction: Oxford University Press, USA.

Bonilla-Priego, M. J., Najera, J. J., \& Font, X. (2011). Environmental management decision-making in certified hotels Journal of Sustainable Tourism, 19(3), 361-382.

Bremner, C. (2009). Sustainable tourism moves slowly in the right direction: Euromonitor International. London.

Bufoni, A. L., Muniz, N. P., \& Ferreira, A. C. D. S. (2009). O Processo de Certificação Socioambiental das Empresas: o Estudo de Caso do Certificado 'Empresa Cidadã'. RAC, 13, 19-38.

Bursztyn, M. A., \& Bursztyn, M. (2006). Desenvolvimento Sustentável: a biografia de um conceito. In E. P. D. V. Nascimento, João Nildo (Orgs.) (Ed.), Economia, Meio Ambiente e Comunicação. Rio de Janeiro: Garamond.

Carlsen, J., Morrison, A., \& Weber, P. (2008). Lifestyle Oriented Small Tourism Firms. Tourism Recreation Research, 33(3), 255-263.

Carrieri, A. D. P., Da Silva, A. R. L., \& Pimentel, T. D. (2009). O Tema da Proteção Ambiental Incorporado nos Discursos da Responsabilidade Social Corporativa. RAC, 13(1), 1-16.

Carroll, A. B., \& Shabana, K. M. (2010). The business case for corporate social responsibility: a review of concepts, research and practice. International Journal of Management Reviews, 12(1), 85-105.

Carvalho, A., \& Alberton, A. (2007). Um estudo em estabelecimentos de hospedagem na Estrada Real/MG: as variáveis social e ambiental. Revista Hospitalidade, 5(1), 31-57.

Dahlstrand, U., \& Biel, A. (1997). Pro-Environmental Habits: Propensity Levels in Behavioral Change1. Journal of Applied Social Psychology, 27(7), 588-601.

Dewhurst, H., \& Thomas, R. (2003). Encouraging sustainable business practices in a non-regulatory environment: a case study of small tourism firms in a UK National Park. Journal of Sustainable Tourism, 11(4), 383-403.

Dewhurst, P., \& Horobin, H. (1998). Small business owners. In R. Thomas (Ed.), The Management of Small Tourism and Hospitality Firms (pp. 19-38). London/NY: Cassell.

Dodds, R., \& Holmes, M. R. (2010). Sustainability in Canadian B\&Bs: comparing the east versus west. International Journal of Tourism Research, 13(5), 482-495.

Farias, J. S., \& Teixeira, R. M. (2002). A pequena e micro empresa e o meio ambiente: a percepção dos empresários com relação aos impactos ambientais. Organizações e Sociedade, 9(23).

Fassin, Y., Van Rossem, A., \& Buelens, M. (2011). Small-Business Owner-Managers' Perceptions of Business Ethics and CSR-Related Concepts. Journal of Business Ethics, 98, 425-453.

Font, X., \& Harris, C. (2004). Rethinking standards from green to sustainable. Annals of Tourism Research, 31(4), 986-1007.

Fuller, T., \& Tian, Y. (2006). Social and Symbolic Capital and Responsible Entrepreneurship: An Empirical Investigation of SME Narratives. Journal of Business Ethics, 67, 287-304.

Garay, L., \& Font, X. (2012). Doing good to do well? Corporate Social Responsibility Reasons, Practices and Impacts in Small and Medium Accommodation Enterprises. International Journal of Hospitality Management, 31(2), 328-336.

Garay, L.; Font, X. (2013). Corporate social 
responsibility in tourism small and medium enterprises evidence from Europe and Latin America. In Tourism Management Perspectives vol, 7, 38-46

Getz, D., \& Carlsen, J. (2000). Characteristics and goals of family and owner-operated businesses in the rural tourism and hospitality sectors. Tourism Management, 21, 547-560.

Griffin, J. J., \& Mahon, J. F. (1997). The corporate social performance and corporate financial performance debate. Business \& Society, 36(1), 5-31.

Hall, M., \& Rusher, K. (2004). Risky lifestyles? Entrepreneurial characteristics of the New Zealand Bed and Breakfast sector. In R. Thomas (Ed.), Small firms in tourism: international perpectives (pp. 8496). UK: Elsevier.

Hart, S. L. (1995). A Natural-Resource-Based View of the Firm. Academy of Management Review, 20(4), 986-1014.

Horobin, H., \& Long, J. (1996). Sustainable tourism: the role of the small firm. International Journal of Hospitality Management, 8(5), 15-19.

Ibge. (2007). Economia do Turismo: análise das atividades características do turismo 2003. Rio de Janeiro.

Inoue, Y., \& Lee, S. (2011). Effects of different dimensions of corporate social responsibility on corporate financial performance in tourism-related industries. Tourism Management, 32(4), 790-804.

IPEA. (2006). A Iniciativa Privada e o Espírito Público: a evolução da ação social das empresas privadas no Brasil. Brasília: IPEA.

Jenkins, H. (2006). Small business champions for corporate social responsibility. Journal of Business Ethics, 67(3), 241-256.

Kang, K. H., Lee, S., \& Huh, C. (2010). Impacts of positive and negative corporate social responsibility activities on company performance in the hospitality industry. International Journal of Hospitality Management, 29(1), 72-82.

Knowles, T., Macmillan, S., Palmer, J., Grabowski, P., \& Hashimoto, A. (1999). The development of environmental initiatives in tourism: responses from the London hotel sector. International Journal Of Tourism Research, 1(4), 255-265.

Kollmuss, A., \& Agyeman, J. (2002). Mind the gap: why do people act environmentally and what are the barriers to pro-environmental behavior? Environmental education research, 8(3), 239-260.

Lee, S., \& Park, S. Y. (2009). Do socially responsible activities help hotels and casinos achieve their financial goals? International Journal of Hospitality Management, 28(1), 105-112.

Lee, S., \& Park, S. Y. (2010). Financial impacts of socially responsible activities on airline companies. Journal of Hospitality \& Tourism Research, 34(2), 185-203.

Lenziardi, R., Mayer, V. F., \& Ferreira, D. A. (2010). O turista se importa? A responsabilidade social e a escolha de um meio de hospedagem. . Paper presented at the Encontro da Associação Nacional dos Programas de Pós-Graduação em Administração, Rio de Janeiro.

Lepoutre, J., \& Heene, A. (2006). Investigating the impact of firm size on small business social responsibility: a critical review. Journal of Business Ethics, 67(3), 257-273.

Molina-Azorín, J. F., Claver-Cortés, E., PereiraMoliner, J., \& Tarí, J. J. (2009). Environmental practices and firm performance: an empirical analysis in the Spanish hotel industry. Journal of Cleaner Production, 17(5), 516-524.

Moretti, S. L. D. A., \& Campanario, M. D. A. (2009). A Produção Intelectual Brasileira em Responsabilidade Social Empresarial - RSE sob a Ótica da Bibliometria. RAC13(Edição especial), 68-86.

Murillo, D., \& Lozano, J. M. (2006). SMEs and CSR: An approach to CSR in their own words. Journal of Business Ethics, 67(3), 227-240.

Peliano, A. M. T. M. (2001). Bondade ou Interesse? como e por que as empresas atuam na área social. Brasília: IPEA.

Randall, D. M., \& Fernandes, M. F. (1991). The social desirability response bias in ethics research. Journal of Business Ethics, 10(11), 805-817.

Revell, A., \& Blackburn, R. (2007). The business case for sustainability? An examination of small firms in the UK's construction and restaurant sectors. Business strategy and the environment, 16(6), 404-420.

Roberts, S., Lawson, R., \& Nicholls, J. (2006). Generating regional-scale improvements in SME corporate responsibility performance: Lessons from responsibility Northwest. Journal of Business Ethics, 67(3), 275-286.

Rodriguez, F. J. G., \& Del Mar Armas Cruz, Y. (2007). Relation between social-environmental responsibility and performance in hotel firms. International Journal of Hospitality Management, 26(4), 824-839.

Salzmann, O., Ionescu-Somers, A., \& Steger, U. (2005a). The Business Case for Corporate Sustainability: Literature Review and Research Options. European Management Journal, 23(1), 27-36.

Salzmann, O., Ionescu-Somers, A., \& Steger, U. (2005b). The Business Case for Corporate Sustainability::: Literature Review and Research Options. European Management Journal, 23(1), 27-36.

Sampaio, A. R., Thomas, R., \& Font, X. (2012). Why are Some Engaged and Not Others? Explaining Environmental Engagement among Small Firms in Tourism. International Journal of Tourism Research, 14(3), 235-249.

Serpa, D. a. F., \& Fourneau, L. F. (2007). Responsabilidade Social Corporativa: Uma Investigação Sobre a Percepção do Consumidor. $\boldsymbol{R} \boldsymbol{A C}, 11(3), 83-103$.

Shaw, G., \& Williams, A. M. (2004). From lifestyle 
consumption to lifestyle production: changing patterns of tourism entrepreneurship. In R. Thomas (Ed.), Small firms in tourism: International perspectives (pp. 99-114). Oxford: Pergamon.

Spence, L., Schmidpeter, R., \& Habisch, A. (2003a). Assessing Social Capital: Small and Medium Sized Enterprises in Germany and the U.K. Journal of Business Ethics, 47, 17-29.

Spence, L. J. (2007). CSR and small business in a European policy context: the five "C" s of CSR and small business research agenda 2007. Business and Society Review, 112(4), 533-552.

Spence, L. J., Schmidpeter, R., \& Habisch, A. (2003b). Assessing social capital: Small and medium sized enterprises in Germany and the UK. Journal of Business Ethics, 47(1), 17-29.

Stabler, M. a. J., Goodal, B. (1997). Environmental awareness action and performance in the guernsey hospitality sector. Tourism Management, 18(1), 19-33.

Souza, F. M. ; Costa, H. A. (2016). Razões que levam micro e pequenas empresas de ecoturismo ao comportamento responsável na Chapada dos Veadeiros GO. Revista Brasileira de Ecoturismo, v. 9, p. 93-111.

Thomas, R. (1998). An introduction to the study of small tourism and hospitality firms. In R. Thomas (Ed.), The management of small tourism and hospitality firms (pp. 1-17). London/NY: Cassell.

Thomas, R. (2004). International perpectives on small firms in tourism: a synthesis. In R. Thomas (Ed.), Small firms in tourism: international perpectives (pp. 1-16). Uk: Elsevier.

Tilley, F. (2000). Small firm environmental ethics: how deep do they go? Business Ethics: A European Review, 9(1), 31-41.

Tzschentke, N., Kirk, D., \& Lynch, P. A. (2004). Reasons for going green in serviced accommodation establishments. International journal of contemporary hospitality management, 16(2), 116-124.

Tzschentke, N., Kirk, D., \& Lynch, P. A. (2008a). Ahead of their time? Barriers to action in green tourism firms. Service Industries Journal, 28(2), 167-178.

Tzschentke, N., Kirk, D., \& Lynch, P. A. (2008b). Going green: Decisional factors in small hospitality operations. International Journal of Hospitality Management, 27(1), 126-133.

Vernon, J., Essex, S., Pinder, D., \& Curry, K. (2003). The 'greening' of tourism micro businesses: outcomes of focus group investigations in South East Cornwall. Business Strategy and the Environment, 12(1), 49-69.

Vinha, V. D. (2003). As empresas e o desenvolvimento sustentável: da eco-eficiência à responsabilidade social corporativa. In P. May, M. C. Lustosa \& V. D. Vinha (Eds.), Economia do Meio Ambiente (pp. 173-195).

Zschiegner, A. K. C. (2011). Leading Change for
Environmental Management Practices in Tourism: the case of SMEs in South West England. PhD thesis, University of Exeter, Exeter.

\section{Contribuição de cada autor na elaboração do artigo}

COSTA: Construção do referencial teórico - literatura nacional; Elaboração e tradução do instrumento de coleta de dados; Organização da coleta de dados; Interpretação dos resultados; Redação final.

FONT: Construção do referencial teórico - literatura internacional; Elaboração do instrumento de coleta de dados; Interpretação dos resultados.

GARAY: Elaboração do instrumento de coleta de dados; Tratamento e análise quantitativa de dados.

JONES: Desenho da pesquisa on-line; Elaboração do instrumento de coleta de dados. 